\title{
THE APPELL HYPERGEOMETRIC FUNCTIONS AND CLASSICAL SEPARABLE MECHANICAL SYSTEMS
}

\author{
Vladimir Dragović
}

\begin{abstract}
A relationship between two old mathematical subjects is observed: the theory of hypergeometric functions and the separability in classical mechanics. Separable potential perturbations of the integrable billiard systems and the Jacobi problem for geodesics on an ellipsoid are expressed through the Appell hypergeometric functions $F_{4}$ of two variables. Even when the number of degrees of freedom increases, if an ellipsoid is symmetric, the number of variables in the hypergeometric functions does not. Wider classes of separable potentials are given by the obtained new formulae automaticaly.
\end{abstract}

\section{Introduction}

Appell introduced four families of hypergeometric functions of two variables in 1880's. Soon, he applied them in a solution of the Tisserand problem in the celestial mechanics. The Appell functions have several other applications, for example in the theory of algebraic equations, algebraic surfaces... The aim of this paper is to point out the relationship between the Appell functions $F_{4}$ and another subject from classical mechanics - separability of variables in the Hamilton - Jacobi equations.

The equation

$$
\lambda V_{x y}+3\left(y V_{x}-x V_{y}\right)+\left(y^{2}-x^{2}\right) V_{x y}+x y\left(V_{x x}-V_{y y}\right)=0,
$$

appeared in Kozlov's paper [1] as a condition on function $V=V(x, y)$ to be an integrable perturbation of certain type for billiard systems inside an ellipse

$$
\frac{x^{2}}{A}+\frac{y^{2}}{B}=1, \quad \lambda=A-B .
$$

This equation is a special case of the Bertrand-Darboux equation [2,3,4]

$$
\begin{gathered}
\left(V_{y y}-V_{x x}\right)\left(-2 a x y-b^{\prime} y-b x+c_{1}\right)+2 V_{x y}\left(a y^{2}-a x^{2}+b y-b^{\prime} x+c-c^{\prime}\right) \\
+V_{x}(6 a y+3 b)+V_{y}\left(-6 a x-3 b^{\prime}\right)=0 .
\end{gathered}
$$

It corresponds to the choise $a=-1 / 2, b=b^{\prime}=c_{1}=0, c-c^{\prime}=-\lambda / 2$. The Bertrand-Darboux equation represents the necessary and suficient condition for a natural mechanical system with two degrees of freedom

$$
H=\frac{1}{2}\left(p_{x}^{2}+p_{y}^{2}\right)+V(x, y)
$$

AMS Subject Classification (1991): Primary 58F05 
to be separable in the elliptical coordinates or some of their degenerations.

The solutions of the equation (1) in a form of the Laurent polynomials in $x, y$ were described in [5]. The starting observation of this paper, that such solutions are simply related to the well-known hypergeometric functions of the Appell type is presented in the section 3 . Such a relation automatically gives a wider class of solutions of the equation (1). But what is more important, it shows the existence of the connection between separability of the classical systems on one hand, and the theory of hypergeometric functions on the other one. The basic references for the Appell functions are $[6,7,8]$. Further, in section 3, the similar formulae for potential perturbations for the Jacobi problem for geodesics on ellipsoid from [9] and for the billiard systems on the surfaces with constant curvature from [10], are given.

In the case of more than two degrees of freedom, the natural generalization for the equation (1) is system (4). The system (4) for the three degrees of freedom was derived in [11] in a connection with the billiard systems inside an ellipsoid in $R^{3}$ and the Laurent polynomial solutions were given. In section 4, we express these solutions for a symmeric ellipsoid through the hypergeometric functions. Surprisingly, we obtain again the Appell hypergeometric functions of two variables. The number of degrees of freedom increased but the number of variables of the hypergeometric functions didn't!

The system

(4)

$$
\begin{aligned}
& \left(a_{i}-a_{r}\right)^{-1}\left(x_{i}^{2} V_{r s}-x_{i} x_{r} V_{i s}\right)=\left(a_{i}-a_{s}\right)^{-1}\left(x_{i}^{2} V_{r s}-x_{i} x_{s} V_{i r}\right) i \neq r \neq s \neq i ; \\
& \left(a_{i}-a_{r}\right)^{-1} x_{i} x_{r}\left(V_{i i}-V_{r r}\right)-\sum_{j \neq i, r}\left(a_{i}-a_{j}\right)^{-1} x_{i} x_{j} V_{j r} \\
& +V_{i r}\left[\sum_{j \neq i, r}\left(a_{i}-a_{j}\right)^{-1} x_{j}^{2}+\left(a_{r}-a_{i}\right)^{-1}\left(x_{i}^{2}-x_{r}^{2}\right)\right]+V_{i r} \\
& +3\left(a_{i}-a_{r}\right)^{-1}\left(x_{r} V_{i}-x_{i} V_{r}\right)=0, \quad i \neq r,
\end{aligned}
$$

where $V_{i}=\partial V / \partial x_{i}$, of $(n-1)\left(\begin{array}{l}n \\ 2\end{array}\right)$ equations was formulated in [12] for arbitrary number of degrees of freedom $n$. In [12] the generalization of the Bertrand-Darboux theorem was proved. According to that theorem, the solutions of the system (4) are potentials separable in generalized elliptic coordinates (see the Theorem after lemma 2 , below).

Some deeper explanation of the connection between the separability in the elliptic coordinates and the Appell hypergeometric functions is not known yet. 


\section{Basic notations}

The function $F_{4}$ is one of the four hypergeometric functions in two variables introduced by Appell $[8,7]$ and defined as a serries:

$$
F_{4}(a, b, c, d ; x, y)=\sum \frac{(a)_{m+n}(b)_{m+n}}{(c)_{m}(d)_{n}} \frac{x^{m}}{m !} \frac{y^{n}}{n !},
$$

where $(a)_{n}$ is a standard Pochhammer symbol:

$$
\begin{aligned}
& (a)_{n}=\frac{\Gamma(a+n)}{\Gamma(a)}=a(a+1) \ldots(a+n-1), \\
& (a)_{0}=1
\end{aligned}
$$

(For example $m !=(1)_{m}$.)

The series $F_{4}$ is covergent for $\sqrt{x}+\sqrt{y} \leq 1$. The functions $F_{4}$ can be continued to the solutions of the equations:

$$
\begin{gathered}
x(1-x) \frac{\partial^{2} F}{\partial x^{2}}-y^{2} \frac{\partial^{2} F}{\partial y^{2}}-2 x y \frac{\partial^{2} F}{\partial x \partial y}+[c-(a+b+1) x] \frac{\partial F}{\partial x} \\
-(a+b+1) y \frac{\partial F}{\partial y}-a b F=0, \\
y(1-y) \frac{\partial^{2} F}{\partial y^{2}}-x^{2} \frac{\partial^{2} F}{\partial x^{2}}-2 x y \frac{\partial^{2} F}{\partial x \partial y}+\left[c^{\prime}-(a+b+1) y\right] \frac{\partial F}{\partial y} \\
-(a+b+1) x \frac{\partial F}{\partial x}-a b F=0,
\end{gathered}
$$

\section{The separable systems with two degrees of freedom}

\section{Billiard inside an ellipse}

Following $[1,5]$ we will start with a billiard system which describes a particle moving freely within an ellipse (2). At the boundary we assume elastic reflections with equal impact and reflection angles. This system is completely integrable and it has an additional integral

$$
K_{1}=\frac{\dot{x}^{2}}{A}+\frac{\dot{y}^{2}}{B}-\frac{(\dot{x} y-\dot{y} x)^{2}}{A B}
$$

We are interested in a potential perturbations $V=V(x, y)$ such that the perturbated system has an integral $\tilde{K}_{1}$ of the form

$$
\tilde{K}_{1}=K_{1}+k_{1}(x, y)
$$

where $k_{1}=k_{1}(x, y)$ depends only on coordinates. This specific condition leeds to the equation (1) on $V$ (see [1]). 
In [5] the Laurent polynomial solutions of the equation (1) were given. The basic set of solutions consists of the functions

$$
\begin{aligned}
V_{k} & =\sum_{i=0}^{k-2}(-1)^{i} \sum_{s=1}^{k-i-1} U_{k i s}(x, y, \lambda, \alpha)+\alpha y^{-2 k}, \quad k \in N, \\
W_{k} & =\sum_{i=0}^{k-2} \sum_{s=1}^{k-i-1}(-1)^{s} U_{k i s}(y, x, \lambda, \alpha)+\alpha x^{-2 k}, \quad k \in N,
\end{aligned}
$$

where

$$
U_{k i s}=\left(\begin{array}{c}
s+i-1 \\
i
\end{array}\right) \frac{[1-(k-i)][2-(k-i)] \ldots[s-(k-i)]}{\lambda^{s+i} s !} \alpha x^{2 s} y^{-2 k+2 i} .
$$

Now, we are going to rewrite the above formulae:

$$
\begin{aligned}
V_{k} & =\sum_{i=0}^{k-2}(-1)^{i} \sum_{s=1}^{k-i-1} U_{k i s}(x, y, \lambda, \alpha)+\alpha y^{-2 k}, \quad k \in N \\
& =\sum_{i=0}^{k-2}(-1)^{i} \sum_{s=1}^{k-i-1} \frac{\Gamma(s+i) \Gamma(s+i-k+1)}{\Gamma(i+1) \Gamma(s) \Gamma(i-k+1) \Gamma(s+1)} x^{2 s} y^{2(i-k)}+y^{-2 k} \\
& =\frac{1}{y^{2 k}}\left(\sum_{i=0}^{k-2} \sum_{s=1}^{k-i-1} \frac{(1)_{s+i-1}(2-k)_{s+i-1}}{i !(1)_{s-1} s !(1-k)_{i}} x^{2 s}\left(-y^{2}\right)^{i}+1\right) \\
& =\frac{1}{y^{2 k}}\left(x^{2} \sum_{i=0}^{k-2} \sum_{s=0}^{k-i-2} \frac{(1)_{s+i}(2-k)_{s+i}}{(2)_{s}(1-k)_{i}} \frac{\left(x^{2}\right)^{s}}{s !} \frac{\left(-y^{2}\right)^{i}}{i !}+1\right) \\
& =\frac{1}{\tilde{y}^{k}}\left(\tilde{x} F_{4}(1 ; 2-k ; 2,1-k, \tilde{x},-\tilde{y})+1\right),
\end{aligned}
$$

where $\tilde{x}=x^{2}, \tilde{y}=-y^{2}$, and $F_{4}$ is the Appell function. We just obtained the simple formula which expresses the potentials $V_{k}$, for $k \in N$ from [5] through the Appell functions. We can use this formula to spread the family of solutions of the equation (1) out of the set of the Laurent polynomials.

Let $V(x, y)=\sum a_{n m} x^{n} y^{m}$. Then the equation (1) reduces to

$$
n m a_{n, m}=(n+m)\left(m a_{n-2, m}-n a_{n, m-2}\right) .
$$

If one of the indices, for example the first one, belongs to $Z$, then $V$ doesn't have essential singularities. Put $a_{0,-2 \gamma}=1$, where $\gamma$ is not necessary an integer.

If we define

$$
a_{\underbrace{2 s+2}_{n}}, \underbrace{2 i-2 \gamma}_{m}=\frac{(-1)^{i}(1)_{s+i}(2-\gamma)_{s+i}}{(2)_{s}(1-\gamma)_{i} s ! i !} .
$$

it can easily be seen that (6) is a solution of the eqation (5). So, let us denote 


$$
V_{\gamma}=\tilde{y}^{-\gamma}\left(\tilde{x} F_{4}(1,2-\gamma, 2,1-\gamma, \tilde{x}, \tilde{y})+1\right)
$$

Then we have

Theorem 1 Every function $V_{\gamma}$ given with (7) and $\gamma \in C$ is a solution of the equation (1).

Mechanical interpretation: with $\gamma \in R^{-}$and the coefficient multiplying $V_{\gamma}$ positive, we have potential barrier along $x$-axis; so we can consider a cut along negative part of $y$-axis.

\section{The Jacobi problem for geodesics on an ellipsoid}

The Jacobi problem for the geodesics on an ellipsoid

$$
\frac{x^{2}}{A}+\frac{y^{2}}{B}+\frac{z^{2}}{C}=1
$$

has an additional integral

$$
K_{1}=\left(\frac{x^{2}}{A^{2}}+\frac{y^{2}}{B^{2}}+\frac{z^{2}}{C^{2}}\right)\left(\frac{\dot{x}^{2}}{A}+\frac{\dot{y}^{2}}{B}+\frac{\dot{z}^{2}}{C}\right) .
$$

The potential perturbations $V=V(x, y, z)$ such that perturbated systems have the integrals of the form

$$
\tilde{K}_{1}=K_{1}+k(x, y, z)
$$

satisfy the following system (see [9])

$$
\begin{aligned}
& \left(\frac{x^{2}}{A^{2}}+\frac{y^{2}}{B^{2}}+\frac{z^{2}}{C^{2}}\right) V_{x y} \frac{A-B}{A B}-3 \frac{y}{B^{2}} \frac{V_{x}}{A}+3 \frac{x}{A^{2}} \frac{V_{y}}{B}+\left(\frac{x^{2}}{A^{3}}-\frac{y^{2}}{B^{3}}\right) V_{x y}+ \\
& +\frac{x y}{A B}\left(\frac{V_{y y}}{A}-\frac{V_{x x}}{B}\right)+\frac{z x}{C A^{2}} V_{z y}-\frac{z y}{C B^{2}} V_{z x}=0 \\
& \left(\frac{x^{2}}{A^{2}}+\frac{y^{2}}{B^{2}}+\frac{z^{2}}{C^{2}}\right) V_{y z} \frac{B-C}{B C}-3 \frac{z}{C^{2}} \frac{V_{y}}{B}+3 \frac{y}{B^{2}} \frac{V_{z}}{C}+\left(\frac{y^{2}}{B^{3}}-\frac{z^{2}}{C^{3}}\right) V_{y z}+ \\
& +\frac{y z}{B C}\left(\frac{V_{z z}}{B}-\frac{V_{y y}}{C}\right)+\frac{x y}{A B^{2}} V_{x z}-\frac{x z}{A C^{2}} V_{x y}=0 \\
& \left(\frac{x^{2}}{A^{2}}+\frac{y^{2}}{B^{2}}+\frac{z^{2}}{C^{2}}\right) V_{z x} \frac{C-A}{A C}-3 \frac{x}{A^{2}} \frac{V_{z}}{C}+3 \frac{z}{C^{2}} \frac{V_{x}}{A}+\left(\frac{z^{2}}{C^{3}}-\frac{x^{2}}{A^{3}}\right) V_{z x}+ \\
& +\frac{x z}{A C}\left(\frac{V_{x x}}{C}-\frac{V_{z z}}{A}\right)+\frac{z y}{B C^{2}} V_{x y}-\frac{y x}{B A^{2}} V_{y z}=0
\end{aligned}
$$

The system (8) replaces in this problem the equation (1). The solutions of the system in the Laurent polynomial form were found in [9]. We can transform them in a following way. 


$$
\begin{aligned}
& V_{l_{0}}(x, y, z)=\sum_{0 \leq k \leq s, k+c \leq l_{0}}(-1)^{s}\left(\begin{array}{c}
s+k-1 \\
k
\end{array}\right)\left(x^{2}\right)^{-l_{0}+k}\left(y^{2}\right)^{s}\left(z^{2}\right)^{l_{0}-(k+s)-1} \\
& \times \frac{c^{s+k}(c-a)^{s}(c-b)^{k} 2^{k+s}\left(-l_{0}+1\right) \ldots\left(-l_{0}+(k+s)\right)}{b^{k} a^{s}(b-a)^{k+s} 2^{s} 2^{k} s !\left(-l_{0}+1\right) \ldots\left(-l_{0}+k\right)}\left(z^{2}\right)^{l_{0}-(k+s)-1} \\
& =\sum \frac{(s+k-1) !\left(-l_{0}+1\right)\left(-l_{0}+2\right)_{s+k-1}\left(z^{2}\right)^{l_{0}}}{k !(s-1) ! s !\left(-l_{0}+1\right)_{k}\left(x^{2}\right)^{l_{0}}}\left[\frac{x^{2} c(a-c)}{z^{2}(b-a) a}\right]^{s}\left[\frac{y^{2} c(c-b)}{z^{2}(b-a) b}\right]^{k} \\
& =\left(-l_{0}+1\right)\left(\frac{z^{2}}{x^{2}}\right)^{l_{0}} \sum \frac{(1)_{s+k-1}\left(-l_{0}+2\right)_{s+k-1}}{(2)_{s-1}\left(-l_{0}+1\right)_{k}} \hat{x}^{s} \hat{y}^{k} \\
& =\left(-l_{0}+1\right)\left(\frac{z^{2}}{x^{2}}\right)^{l_{0}} F_{4}\left(1 ;-l_{0}+2 ; 2,-l_{0}+1, \hat{x}, \hat{y}\right),
\end{aligned}
$$

where

$$
\frac{x^{2} c(a-c)}{z^{2}(b-a) a}=\hat{x}, \quad \frac{y^{2} c(c-b)}{z^{2}(b-a) b}=\hat{y}
$$

In the above formulae $l_{0}$ was an integer. We have strightforward generalization:

Theorem 2. For every $\gamma \in C$ the function

$$
V_{\gamma}=(-\gamma+1)\left(\frac{z^{2}}{x^{2}}\right)^{\gamma} F_{4}(1 ;-\gamma+2 ; 2,-\gamma+1, \hat{x}, \hat{y})
$$

is a solution of the system (8).

\section{Billiard systems on the constant curvature surfaces}

Potential perturbations of the billiard systems on the constant curvature surfaces were analysed in [10]. Following the notation from [10], let the billiard $D_{S}$ be a subset of the surface $\Sigma_{S}$, of the curvature $S=+1$ or $S=-1$, bounded with the quadric $Q_{S}$, where

$$
\begin{aligned}
& \Sigma_{+}=\left\{r=(x, y, z) \in R^{3} \mid\langle r, r\rangle_{+}=1\right\},\left\langle r_{1}, r_{2}\right\rangle_{+}=x_{1} y_{1}+x_{2} y_{2}+x_{3} y_{3} ; \\
& \Sigma_{-}=\left\{r=(x, y, z) \in R^{3} \mid\langle r, r\rangle_{-}=-1, z>0\right\},\left\langle r_{1}, r_{2}\right\rangle_{-}=x_{1} y_{1}+x_{2} y_{2}-x_{3} y_{3} ; \\
& Q_{S}=\Sigma_{S} \cap\left\{r \in R^{3} \mid\langle Q r, r\rangle_{s}=0\right\} \neq \emptyset, Q=\operatorname{diag}\left(\frac{1}{A}, \frac{1}{B}, \frac{1}{C}\right) .
\end{aligned}
$$

Then the billiard system has the integral

$$
K=\frac{(\dot{x} y-\dot{y} x)^{2}}{A B}+S \frac{(\dot{x} z-\dot{z} x)^{2}}{A C}+S \frac{(\dot{z} y-\dot{y} z)^{2}}{B C}
$$

As before we are looking for potentials $V=V(x, y, z)$ such that the pertubated system has an integral of the form

$$
\tilde{K}=K+k(x, y, z)
$$


In this case the condition is given by the system [10]:

$$
\begin{gathered}
3 C y V_{x}-3 C x V_{y}+V_{x y}\left(C\left(y^{2}-x^{2}\right)+K z^{2}(B-A)\right) \\
+c x y V_{x x}-c x y V_{y y}+a z y V_{z x}-b z x V_{z y}=0, \\
3 B z V_{x}-K 3 B x V_{z}+V_{x z}\left(B\left(z^{2}-K x^{2}\right)+K y^{2}(C-A)\right) \\
+B z x V_{x x}-K B z x V_{z z}+A z y V_{x y}-K C y x V_{y z}=0, \\
3 A z V_{y}-K 3 A y V_{z}+V_{y z}\left(A\left(z^{2}-K y^{2}\right)+K x^{2}(C-B)\right) \\
+A z y V_{y y}-K A z y V_{z z}+B z x V_{x y}-K C x y V_{x z}=0 .
\end{gathered}
$$

Starting from the solutions from $[10]$

$$
V_{l_{0}}=\frac{1}{z^{2 l_{0}}} \sum_{\substack{0 \leq k \leq l_{0}-1 \\ 0 \leq m \leq l_{0}-k-1}} a_{m, k} x^{2 m} y^{2 l_{0}-2-2 k-2 m} z^{2 k},
$$

where

$$
a_{m, k}=K^{l_{0}-k-1}\left(\frac{C-B}{C-A}\right)^{m}\left(\begin{array}{c}
l_{0}-k-1 \\
m
\end{array}\right)\left(\begin{array}{c}
k+m-1 \\
k
\end{array}\right)\left(\frac{A-B}{C-A}\right)^{k},
$$

we come to

Theorem 3. The functions

$$
V_{\gamma}=\hat{y}^{-\gamma}\left(x^{2} F_{4}(1,2-\gamma, 2,1-\gamma, \hat{x}, \hat{y})+1\right),
$$

where

$$
\frac{x^{2}(B-C)}{y^{2}(C-A)}=\hat{x}, \quad K \frac{z^{2}(A-B)}{y^{2}(C-A)}=\hat{y}
$$

are solutions of the system (10), for $\gamma \in C$.

\section{More than two degrees of freedom}

In the previous section we saw that the integrable perturbations of separable systems with two degrees of freedom led to the hypergeometric functions of two variables. Now, one can expect that in a case of more than two degrees of freedom, the integrable potentials are connected with the hypergeometric functions again, but with more than two variables. We will consider the billiard system inside an ellipsoid in $R^{3}$, and we will see that corresponding potential perturbations are still related to the Appell function $F_{4}$ of two variables, if the ellipsoid is symmetric.

\section{Billiards inside an symmetric ellipsoid in $R^{3}$}

Let us consider the billiard system within an ellipsoid in $R^{3}$

$$
\frac{x^{2}}{A}+\frac{y^{2}}{B}+\frac{z^{2}}{C}=1
$$


Potential perturbations $W=W(x, y, z)$ of such systems in a form of the Laurent polynomials were calculated in [11]. They satisfy the system (4) for $n=3$.

$$
W_{l_{0}}=\frac{1}{z^{2 l_{0}}} \sum_{0 \leq m+n+k<l_{0}} \frac{\left(l_{0}-k-1\right) !(-1)^{n}}{m ! n !\left(l_{0}-1-k-m-n\right) !} \frac{P_{m, n}^{k}(\beta, \gamma)}{\gamma^{m+k} \beta^{n+k}} x^{2 m} y^{2 n} z^{2 k},
$$

where

$$
P_{m, n}^{k}(\beta, \gamma)=\sum_{i=0}^{k}\left(\begin{array}{c}
m+k-1-i \\
k-i
\end{array}\right) \cdot\left(\begin{array}{c}
n+i-1 \\
i
\end{array}\right)(-1)^{i} \beta^{k-i} \gamma^{i} .
$$

and $\beta=B-C, \gamma=C-A$.

The symmetric case $A=B$ corresponds to the condition $\gamma+\beta=0$.

Lemma 1. If $\gamma+\beta=0$ then we have

$$
P_{m, n}^{k}(\beta,-\beta)=\left(\begin{array}{c}
k+m+n-1 \\
k
\end{array}\right) \beta^{k} .
$$

Proof.

$$
\begin{aligned}
P_{m, n}^{k}(\beta,-\beta) & =\beta^{k} \sum_{i=0}^{k}\left(\begin{array}{c}
m+k-i-1 \\
k-i
\end{array}\right)\left(\begin{array}{c}
n+i-1 \\
i
\end{array}\right) \\
& =\beta^{k} \sum_{i=0}^{k}\left(\begin{array}{c}
k-i+m-1 \\
k-i
\end{array}\right)\left(\begin{array}{c}
i+n-1 \\
i
\end{array}\right)=\left(\begin{array}{c}
k+m+n-1 \\
k
\end{array}\right) \beta^{k} .
\end{aligned}
$$

By putting (12) into (11) we get, using $\gamma=-\beta$

$$
\begin{aligned}
& W_{l_{0}}=C \frac{1}{\hat{z}^{l_{0}}} \sum_{m+n+k=1}^{l_{0}} \frac{\left(l_{0}-1\right) \ldots\left(l_{0}-m-n-k\right)(m+n+k-1) !(-1)^{n}}{\left(l_{0}-1\right) \ldots\left(l_{0}-k\right)(m+n-1) !} \frac{\hat{x}^{m}}{m !} \frac{\hat{y}^{n}}{n !} \frac{\hat{z}^{k}}{k !} \\
& =\frac{C}{(\hat{z})^{l_{0}}}\left[\sum_{m+n+k=1}^{l_{0}} \frac{\left(-l_{0}+2\right) \ldots\left(-l_{0}+m+n+k\right)(1)_{m+n+k-1}}{\left(-l_{0}+1\right) \ldots\left(-l_{0}+k\right)(-1)^{-m}(1)_{m+n-1}} \frac{\hat{x}^{m}}{m !} \frac{\hat{y}^{n}}{n !} \frac{\hat{z}^{k}}{k !}+1\right]
\end{aligned}
$$

where $\hat{x}=\frac{x^{2}}{\gamma}, \hat{y}=\frac{y^{2}}{\beta}, \hat{z}=\frac{z^{2}}{\gamma}$.

$$
\begin{aligned}
& W_{l_{0}}=C \cdot \hat{z}^{-l_{0}}\left(\sum_{m+n+k=1}^{l_{0}} \frac{(1)_{m+n+k-1}\left(2-l_{0}\right)_{m+n+k-1}(-1)^{m}}{(1)_{m+n-1}\left(1-l_{0}\right)_{k}} \frac{\hat{x}^{m}}{m !} \frac{\hat{y}^{n}}{n !} \frac{\hat{z}^{k}}{k !}+1\right) \\
& =C \cdot \hat{z}^{-l_{0}}\left(\sum_{m+n+k=1}^{l_{0}} \frac{(1)_{m+n+k-1}\left(2-l_{0}\right)_{m+n+k-1}(-1)^{m}(m+n) !}{(2)_{m+n-1}\left(1-l_{0}\right)_{k}(m+n-1) !} \frac{\hat{x}^{m}}{m !} \frac{\hat{y}^{n}}{n !} \frac{\hat{z}^{k}}{k !}+1\right) \\
& =C \cdot \hat{z}^{-l_{0}}\left(\sum_{m+n+k=1}^{l_{0}} \frac{(1)_{m+n+k-1}\left(2-l_{0}\right)_{m+n+k-1}}{(2)_{m+n-1}\left(1-l_{0}\right)_{k}} \frac{(-\hat{x}+\hat{y})^{m+n}}{(m+n-1) !} \frac{\hat{z}^{k}}{k !}+1\right) \\
& =C \cdot \hat{z}^{-l_{0}}\left[(-\hat{x}+\hat{y}) \sum_{0 \leq s+k<l_{0}} \frac{(1)_{s+k}\left(2-l_{0}\right)_{s+k}}{(2)_{s}\left(1-l_{0}\right)_{k}} \frac{(-\hat{x}+\hat{y})^{s}}{s !} \frac{\hat{z}^{k}}{k !}+1\right],
\end{aligned}
$$


where $C=\left(1-l_{0}\right) \gamma^{-l_{0}}, m+n-1=s$.

Theorem 4. Generalizations of the integrable potential perturbations from [11] in the symmetric case are given by:

$$
W_{l_{0}}=(\hat{z})^{-l_{0}}\left[(-\hat{x}+\hat{y}) F_{4}\left(1,2-l_{0} ; 2,1-l_{0} ;-\hat{x}+\hat{y}, \hat{z}\right)+1\right],
$$

where $l_{0} \in C$.

\section{The case of general dimension}

We consider billiard system in $R^{n}$ within an ellipsoid

$$
\frac{x_{1}^{2}}{a_{1}}+\cdots+\frac{x_{n}^{2}}{a_{n}}=1 .
$$

For $n \geq 3$ we start from a separable system with $n$ integrals $K_{1}, \ldots, K_{n}$ which are mutualy in involution, where $K_{n}=H$ is a Hamiltonian and

$$
K_{i}=\sum_{j \neq i} \frac{\left(\dot{x}_{i} x_{j}-\dot{x}_{j} x_{i}\right)^{2}}{a_{i}-a_{j}}, \quad i=1, \ldots, n-1 .
$$

Then we are interested in potential perturbations $k_{1}, \ldots, k_{n}$, where $k_{i}=k_{i}\left(x_{1}, \ldots, x_{n}\right)$ depend only on coordinates and $V=k_{n}$. The conditions

$$
\left\{\tilde{K}_{n}, \tilde{K}_{i}\right\}=0, \quad i=1, \ldots, n-1,
$$

where

$$
\tilde{K}_{i}=K_{i}+k_{i}, \quad i=1, \ldots, n,
$$

are equivalent to the system (4). Nontrivial (and maybe unexpected) fact is that new integrals commute between themselves.

Lemma 2 From (13) and (14), it follows that

$$
\left\{\tilde{K}_{j}, \tilde{K}_{i}\right\}=0, \quad i, j=1, \ldots, n-1 .
$$

This was checked by direct calculation for $n=3$ in [11], but in general case it follows from the generalized Bertrand- Darboux theorem proved in [12]:

Generalized Bertrand-Darboux Theorem [12] For a natural Hamiltonian system with a Hamiltonian

$$
H=\frac{1}{2} \sum_{i=1}^{n} p_{i}^{2}+V(x)
$$

the following three conditions are equivalent: 
(a) It has $n-1$ global, indipendent, involutive integrals of the form (15).

(b) The potential $V$ satisfies the system (4).

(c) The Hamilton - Jacobi equation for $H$ is separable in generalized elliptic coordinates $\left(u_{1}, \ldots, u_{n}\right)$ given by

$$
1+\sum_{i=1}^{n} \frac{x_{i}}{z-a_{i}}=\frac{\prod_{j=1}^{n}\left(z-u_{j}\right)}{\prod_{k=1}^{n}\left(z-\alpha_{k}\right)}
$$

Theorem 5 Integrable potential perturbations of the billiard systems inside a symetric ellipsoid in $R^{n}$ for arbitrary $n \in N$ are given with

$$
V_{k}^{n}=\left(\hat{x}_{n}\right)^{-k}\left[\left(\hat{x}_{1}+\cdots+\hat{x}_{n-1}\right) F_{4}\left(1,2-k ; 2,1-k ; \hat{x}_{1}+\cdots+\hat{x}_{n-1}, \hat{x}_{n}\right)+1\right]
$$

for any $k \in C$.

\section{Conclusion}

If we denote in (11) $-\beta / \gamma$ as $q$, then, theorem 4 shows that the potentials $W_{l_{0}}$ are certain deformations of the (Appell) hypergeometric functions. The analysis of this deformation and comparison to the known $q$-deformations and generaisations of the hypergeometric functions multivariable ([6]) remains as an interesting problem.

Potential perturbations of the classical nonholonomic rigid body problems are described in [13]. It looks that they are also connected with the hypergeometric functions.

From geometric point of view, it is well known that the billiard systems within an ellipse are closely related to the Poncelet and the Cayley theorem [14-16]. So, the Appell hypergeometric functions define natural deformations of these classical projective geometry settings.

Acknowledgment This research was partialy done during the author's visit to the Mathematical Department of the Kyoto University as a Matsumae International

Foundation Fellow. It is a great pleasure to thank professor M. Jimbo for warm hospitality and A.Yu. Orlov for valuable discussions.

\section{References}

[1] Kozlov V.V.: Some integrable generalizations of the Jacobi problem for the geodesics on the ellipsoid (in Russian), Prikl. Mat. Mekh., Vol. 59, No.1, 3-9, (1995.)

[2] Whittaker E.T.: A treatise on the analitical dynamics of particles and rigid bodies, thierd edition, Cambridge, the University press (1927)

[3] Bertrand J., Journ.de Math., Vol. 17, p. 121, (1852)

[4] Darboux G.: Archives Neerlandaises (2), Vol. 6, p. 371 (1901)

[5] Dragovic V.: On integrable potentials of billiard within ellipse (in Russian), Prikl. Mat. Mekh., Vol. 62, (1998.), No. 1, 166-169. 
[6] Vilenkin N. Ja. and Klimyk A. U.:Representation of Lie groups and special functions, Recent Advances, Kluwer Academic Rublishers, p. 497 (1995)

[7] Appell P. and Kampe de Feriet J.: Fonctions hypergeometriques et hyperspheriques. Polynomes d'Hermite, Gauthier Villars, Paris (1926)

[8] Appell P. Sur les fonctions hypergeometriques de deux variables et sur des equations lineaires aux derivees partielles, Comptes Rendus, v.90, p. 296 (1880)

[9] Dragovic V.: On integrable perturbations of the Jacobi problem for the geodesics on the ellipsoid, J. of Phys. A, Math. and General, Vol. 29, L317-L321, (1996.)

[10] Jovanovic B.: Integrable perturbation of billiards on constant curvature surfaces, Phys. Lett. A, Vol. 231, 353-358, (1997.)

[11] Dragovic V., Jovanovic B.: On integrable potential perturbations of billiard system within ellipsoid, J. of Math. Phys., Vol. 38, (1997.)

[12] Marshall I., Wojciechowski S.: When is a Hamiltonian system Separable?

[13] Dragović V., Gajić B., Jovanović B.:Generalizations of classical integrable nonholonomic rigid bodu sustems, J. Phys A: Math. Gen., Vol. 31, 9861-9869 (1998)

[14] H. Lebesgue Les coniques, Gauthier-Villars, Paris, (1942), 115-149.

[15] P. Griffiths, J. Harris On Cayley's explicit solution to Poncelet's porism, L'Enseignement mathem. 24, (1978), 31-40.

[16] Dragović V., Radnović M. On periodical trajectories of the billiard systems within an ellipsoid in $R^{d}$ and generalized Cayley's condition J. of Math. Phys.,Vol. 39, No. 11, (1998)

Mathematical Institute SANU, Kneza Mihaila 35, 11000 Belgrade, Yugoslavia

E-mail address: vladad@ mi.sanu.ac.yu 